\title{
Postmortem magnetic resonance imaging of the heart ex situ: development of technical protocols
}

\author{
C. Bruguier • C. Egger • J. P. Vallée • J. Grimm • \\ X. Boulanger • C. Jackowski $\cdot$ P. Mangin $\cdot$ S. Grabherr
}

Received: 7 May 2014 / Accepted: 30 July 2014 / Published online: 10 August 2014

(C) Springer-Verlag Berlin Heidelberg 2014

\begin{abstract}
Postmortem MRI (PMMR) examinations are seldom performed in legal medicine due to long examination times, unfamiliarity with the technique, and high costs. Furthermore, it is difficult to obtain access to an MRI device used for patients in clinical settings to image an entire human body. An alternative is available: ex situ organ examination. To our knowledge, there is no standardized protocol that includes ex situ organ preparation and scanning parameters for postmortem MRI. Thus, our objective was to develop a standard procedure for ex situ heart PMMR examinations. We also tested the oily contrast agent Angiofil ${ }^{\circledR}$ commonly used for PMCT angiography, for its applicability in MRI. We worked with a 3 Tesla MRI device and 32-channel head coils. Twelve porcine hearts were used to test different materials to find the best way to prepare and place organs in the device and to test scanning parameters. For coronary MR angiography, we tested different mixtures of Angiofil ${ }^{\circledR}$ and different injection materials. In a second step, 17 human hearts were examined
\end{abstract}

C. Bruguier $(\triangle) \cdot$ C. Egger $\cdot$ J. Grimm $\cdot$ P. Mangin $\cdot$ S. Grabherr University Center of Legal Medicine, Lausanne-Geneva, University of Lausanne, Rue du Bugnon 21, 1011 Lausanne, Switzerland e-mail: christine.bruguier@chuv.ch

C. Bruguier $\cdot$ J. Grimm $\cdot$ X. Boulanger

Department of Diagnostic and Interventional Radiology, University

Hospital Lausanne, Rue du Bugnon 46, 1011 Lausanne, Switzerland

C. Egger $\cdot$ P. Mangin

University Center of Legal Medicine, Lausanne-Geneva, University of Geneva, Rue. Michel-Servet 1, 1211 Geneva 4, Switzerland

J. P. Vallée

Department of Radiology, Geneva University Hospitals and University of Geneva, Rue. Grabrielle Perret-Gentil 4, 1205 Geneva, Switzerland

C. Jackowski

Center for Forensic Imaging, Institute of Forensic Medicine,

University of Bern, Buehlstr. 20, 3012 Bern, Switzerland to test the procedure and its applicability to human organs. We established two standardized protocols: one for preparation of the heart and another for scanning parameters based on experience in clinical practice. The established protocols enabled a standardized technical procedure with comparable radiological images, allowing for easy radiological reading. The performance of coronary MR angiography enabled detailed coronary assessment and revealed the utility of Angiofil ${ }^{\circledR}$ as a contrast agent for PMMR. Our simple, reproducible method for performing heart examinations ex situ yields high quality images and visualization of the coronary arteries.

Keywords Postmortem MRI · Contrast agent · Coronary MR angiography $\cdot$ Ex situ heart $\cdot$ Protocol

\section{Introduction}

Multidetector computed tomography (MDCT) and magnetic resonance imaging (MRI) are important investigation tools that are frequently used in forensic medicine. Postmortem computed tomography (PMCT) has become more widespread, and several institutes of legal medicine now own an MDCT unit with which to examine the deceased. Although PMCT can provide information about the skeletal system, it is less than optimal for examining soft tissue, such as inner organs and the vascular system [1]. To overcome this weakness, contrast agent can be injected to allow for better contrast of organs and visualization of vessels. In the University Center of Legal Medicine Lausanne-Geneva, multiphase postmortem computed tomography angiography (MPMCTA) is routinely performed with the oily contrast agent Angiofil ${ }^{\circledR}$. This technique provides highly detailed images of the vascular system [2-4] of the head, thorax, and abdomen. It permits detailed examination of, for example, the coronary arteries, which is especially important in cases of ischemic heart disease [5]. A 
recently published study [1] showed that by performing MPMCTA, the sensitivity of PMCT could be increased from $65 \%$ (PMCT only) to $80.9 \%$ of detectable findings. By comparison, conventional autopsy was able to detect $83.1 \%$ of all findings. However, the most important weakness of the radiological technique compared to autopsy was the low sensitivity for detecting findings in the parenchyma of inner organs (79.1 \% for MPMCTA vs. $97.7 \%$ for autopsy). This weakness of postmortem imaging could potentially be overcome by using MRI, which has a higher sensitivity for softtissue exploration compared to MDCT [6, 7].

Several studies have investigated cardiac parenchyma with postmortem magnetic resonance imaging (PMMR) and had promising results [8-12]. However, although some authors have proposed an interpretation of postmortem changes in PMMR and guidelines for cardiac PMMR [13-15], there are no guidelines for investigating the heart ex situ with PMMR. Another limitation of PMMR is that few institutes for legal medicine own an MRI device, and access to this imaging modality in a clinical center is not an easy matter. PMMR is therefore often performed at the end of the daily program, between patients, or during the weekend, and not necessarily with specialized technical teams (radiographer and radiologist). Moreover, MRI requires stringent preparation, ensuring that no ferromagnetic objects are hidden in or around the body, and use of a special MRI-compatible carriage. The difficulty is increased when the institute of legal medicine is located far from a radiology department.

Cardiac incidences are a common cause of death encountered in forensic medicine [16]. Postmortem imaging, especially PMMR, could be of great interest as a complement to standard autopsy and histological investigations [12, 14, 17]. Thus, despite the constraints of this imaging modality, our objective was to develop a protocol that would enable the forensic pathologist to perform a PMMR examination of cardiac parenchyma with optimized efficiency with respect to time and technical aspects.

The purpose of our study was as follows: (1) to develop a protocol for the preparation of ex situ hearts, including a coronary MR angiography for PMMR, that would provide sufficient imaging data for a systematic examination of coronary arteries and cardiac parenchyma in cases of suspected cardiac death, without impeding autopsy and (2) to investigate if the oily contrast agent Angiofil ${ }^{\circledR}$, which is used for MPMCTA, can be used with PMMR imaging to perform a heart-focused examination after a previous whole-body MPMCTA.

\section{Materials and methods}

The most important criterion for this study was that the whole procedure, which comprised two protocols (preparation of ex situ hearts and PMMR acquisitions), had to be reproducible. We considered technical and practical limitations due to the specific features of the MRI device and the time available for acquisition (time location of the MRI device and autopsy duration). This study was performed using two different models: first an animal model, then a human model.

Animal heart model

The technical procedure was developed on fresh porcine hearts (exact postmortem delay unknown), which were obtained from a local butcher $(n=12)$.

Six porcine hearts were used to test ex situ organ preparation, materials, positioning, spatial localization, and MRI sequences. Another six porcine hearts were used to train a team of forensic radiographers to prepare the heart in such a way as to ensure reproducibility. Porcine hearts were chosen because of their similarity to human hearts with respect to anatomy, shape, and weight. The preparation technique consisted of careful dissection around the proximal first millimeters of the coronary arteries (right coronary artery and common trunk of the left coronary artery). The vessels were then injected with contrast agent using different mixtures of the oily contrast agent Angiofil ${ }^{\circledR}$ (Fumedica, Muri, Switzerland) with paraffin oil and different injection materials (different types of catheters, needles, and syringes). Finally, the heart was correctly positioned inside containers to enable spatial orientation.

\section{Imaging}

Scans were performed on a 3 Tesla MRI scanner (Trio; Siemens, Erlangen, Germany) dedicated to clinical and functional brain imaging researches, and we used a 32-channel head coil. Ex situ heart MRI examinations require a container to maintain the shape of the heart and to avoid collapse of its cavities or compression of its underlying structures. Different techniques of positioning in the magnet were tested, as were different container systems for fixing the heart in the correct position. We identified massive movement artifacts during data acquisition, due to vibrations of the table. Therefore, we tested the ability of various materials to weight the containers (e.g., sand, decorative sand, and flour).

Human heart model

Once the technical approach had been tested and practiced on the animal heart model, we applied it to human hearts. This part of the study adhered to international and local ethical standards and regulations and was approved by the local ethics committee. We investigated 17 human hearts that had been extracted during conventional medicolegal autopsy following the local standard autopsy procedure (extraction of the 
heart and origins of the aorta, pulmonary artery, and veins after opening the pericardium). The postmortem delay varied between 12 and $48 \mathrm{~h}$. Preparation of the heart, injection of coronary arteries, and MRI data acquisition were then performed within the next $5 \mathrm{~h}$. Once the data acquisition was complete, the heart was returned to the forensic pathologist in charge of the case so that he or she could perform a standard autopsy dissection of the organ and return it to the body before closing the body.

\section{Results}

We established two protocols in order to obtain the final procedure. The first was a standardized protocol for preparation of the ex situ heart for PMMR, and the other was for scanning parameters used in MRI acquisition based on clinical cardiac MRI guidelines. We were also able to evaluate the MRI compatibility of Angiofil ${ }^{\circledR}$.

\section{Protocol for preparation of the heart}

This protocol included preparation of the coronary arteries, preparation of the ventricles, and ensuring correct spatial localization of the ex situ organ. The heart was extracted from the body, together with about $3 \mathrm{~cm}$ of both the ascending thoracic aorta and the pulmonary trunk. These two major vessels were separated from each other by careful dissection down to their proximal portion, which provided access to the coronary ostia in the ascending aorta. Debridement of the proximal first millimeters of each coronary artery was performed to enable ligation close to the ostia after they were filled with contrast agent (Fig. 1a).

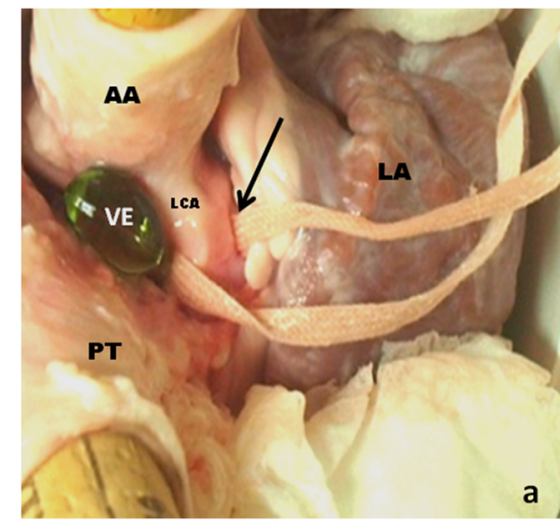

Fig. 1 Preparation of ex situ hearts. a Careful debridement of the proximal millimeters of each coronary artery was required to introduce a bond under each vessel (black arrow), which enabled their ligature close to the ostia. $A A$ ascending aorta, $P T$ pulmonary trunk, $L A$ left auricle, $L C A$ left coronary artery; $V E$, capsule of vitamin $\mathrm{E}$ that was placed between the
The right coronary artery and the left coronary artery with its major branches (the circumflex artery and left anterior descending artery) were filled with contrast agent by means of the cardiac catheter introducer $4 \mathrm{~F}$ (Introducer II; Terumo Corporation, Tokyo, Japan). We used a $50 \%$ dilution of the oily contrast agent Angiofil ${ }^{\circledR}$ with paraffinum perliquidum (Hänseler AG, Switzerland) that were carefully injected manually. Ligature of the coronary arteries was performed immediately after injection of contrast agent to ensure that it did not flow out of the vessels. Two small balls of modeling clay were made and placed in cellophane paper or in the finger of a latex glove; they were then placed in each of the two ventricles to separate the walls (Fig. 1b).

To avoid movement artifacts, a system of two containers made of glass was chosen: a small one in which the heart was positioned vertically and a larger one containing the small glass. The space between the two glasses was filled with flour (Fig. 2a) to weight the system.

For better spatial orientation, a simple cork stopper was introduced into the pulmonary trunk and a cork stopper containing a capsule of vitamin $\mathrm{E}$ was placed in the thoracic ascending aorta (Fig. 2b). The capsule of vitamin E is oily and produced an MRI signal comparable to the oily contrast agent, facilitating localization of the anatomical structures of the heart (Fig. 2c). A second capsule of vitamin E was placed medially at the anterior side of the heart inside the small glass.

\section{Protocol for scanning parameters}

MRI examinations were performed with a 32-channel head coil. The position of the heart in the coil was standardized in the vertical position with the anterior face of the ventricles towards the entrance of the magnet.

Our protocol contained T1-weighted, fat-saturation T1weighted, T2-weighted, and fat-saturation T2-weighted

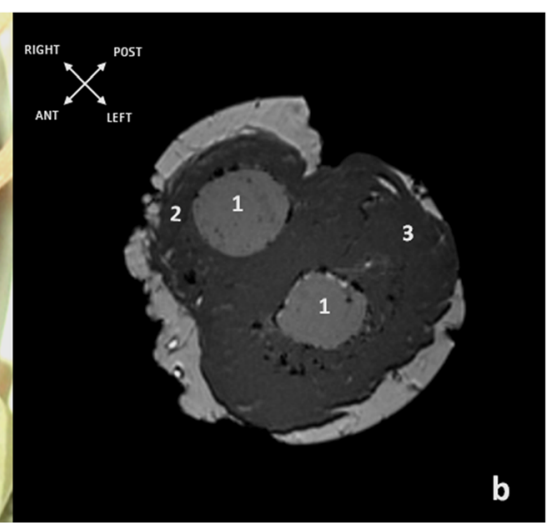

thoracic ascending aorta and the pulmonary trunk for the first tests. However, for the following examinations, it was placed in the cork stopper in the thoracic ascending aorta. b Modeling clay (1) in the right (2) and left (3) ventricles to prevent collapse of the cavities.

$A N T$, anterior; POST, posterior 


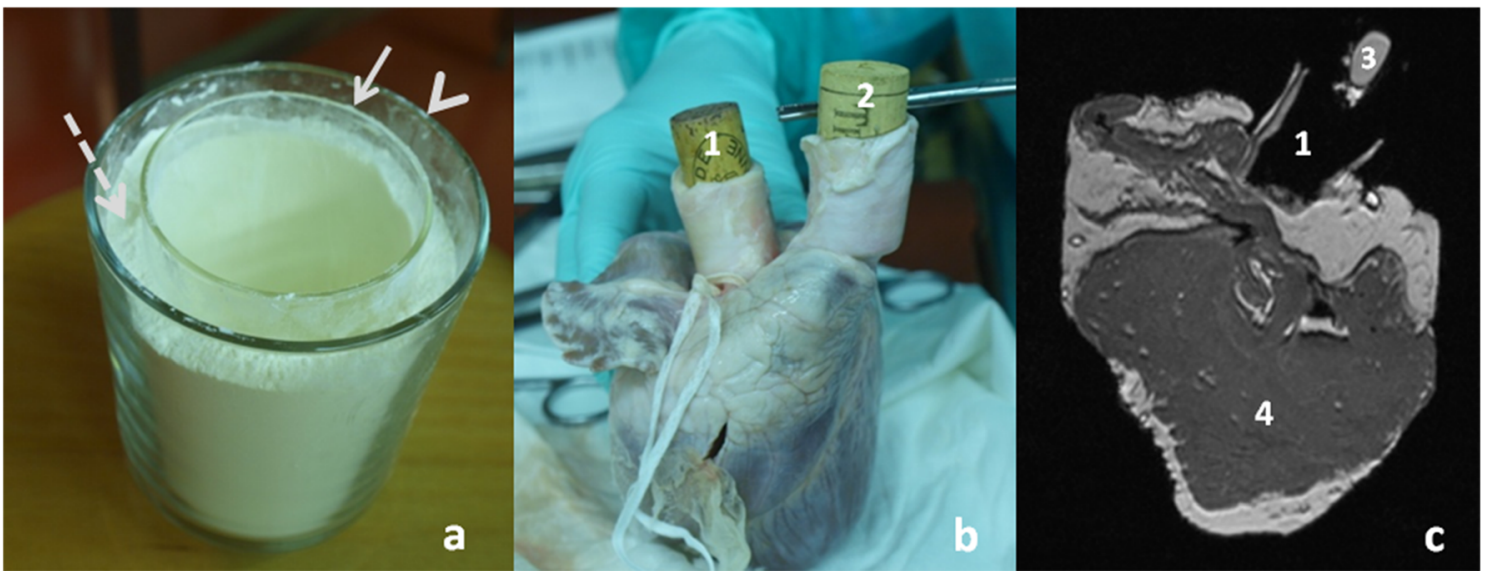

Fig. 2 Preparation of ex situ hearts and spatial localization. a For PMMR examinations, hearts were maintained in a system of two containers made of glass: a small glass (arrow) inside a larger, heavy glass (arrowhead). The hearts were introduced to the system in a vertical position. Space between the two containers was filled with flour (dashed arrow). b A cork

sequences, as well as some acquisitions in 3D (T1 3D and T2 Fat/Sat 3D). All of the sequences had to be performed in a predetermined acquisition time of $1 \mathrm{~h}$. The MRI parameters that we used are shown in Table 1. The sequences were acquired in the short axis of the left ventricle.

\section{Contrast agent}

MRI examinations using Angiofil ${ }^{\circledR}$ was performed with different sequences to ensure the absence of artifacts. In our setup, the contrast agent did not generate any artifacts at MRI and allowed contrasting the arterial lumen and therefore the evaluation of coronary arteries (Fig. 3).

\section{Discussion}

Our starting point was the ex situ macroscopic examination of the heart during autopsy $[18,19]$. MRI examination had to be performed as soon as the heart was eviscerated, before its

Table 1 Sequence parameters for PMMR

\begin{tabular}{lllll}
\hline Sequence & $\begin{array}{l}\text { TR } \\
(\mathrm{ms})\end{array}$ & TE $(\mathrm{ms})$ & $\begin{array}{l}\text { Slice thickness } \\
(\mathrm{mm})\end{array}$ & $\begin{array}{l}\text { DFOV } \\
(\mathrm{cm})\end{array}$ \\
\hline T1 3D & 1,900 & 2.4 & 0.6 & $16 \times 16$ \\
T2 fat/sat 3D & 2,800 & 352 & 0.9 & $20 \times 20$ \\
T1 fat/sat & 704 & 8.8 & 2 & $20 \times 20$ \\
T2 & 6,100 & 105 & 2 & $20 \times 20$ \\
T2 fat/sat & 7,000 & 105 & 2 & $20 \times 20$ \\
T2 & 13,280 & $96 ; 105 ; 201$ & 2.5 & $20 \times 20$ \\
\hline
\end{tabular}

$T R$ time of repetition, $T E$ time of echo, $F O V$ field of view, $m s$ milliseconds, $\mathrm{mm}$ millimeter, $\mathrm{cm}$ centimeter stopper containing a capsule of vitamin $\mathrm{E}$ was placed in the thoracic ascending aorta (1), and a second cork stopper (with no vitamin $\mathrm{E}$ capsule) was placed in the pulmonary trunk (2). c MRI image of the orientation system with the ascending aorta (1), the capsule of vitamin $\mathrm{E}$ (3) in the cork stopper, and the left ventricle (4)

dissection by the forensic pathologist. Moreover, because coronary artery disease is a frequent cause of death and because localization of lesions in the coronary arteries corresponds to the localization of the damaged myocardium [20], the protocol needed to allow detailed coronary artery assessment.

Clinical experience of cardiac MRI with in situ heart examination was not sufficient to develop postmortem ex situ cardiac MRI examination. Special preparation of the heart and appropriate injection technique to completely fill the coronary arteries with contrast agent were needed.

For ex situ cardiac PMMR, the selection of the equipment is important. Firstly, material must be MRI-compatible, which means that it does not contain any metallic or ferromagnetic component that could interfere with the magnetic field. Secondly, the entire disposal has to fit in the 32-channel head coil and be well stabilized to limit movement artifacts.

\section{Choice of container}

For the performance of our first postmortem MRI examination on a human ex situ heart, we used a predefined protocol with sequences usually used in clinical practice to enable a radiologist to read the obtained images. The prepared heart was put in a simple and thin plastic recipient, and placed in the MRI device. Quality of the first images was satisfying for the majority of sequences but for some of them, we observed movement artifacts (Fig. 4a). We had to understand why such artifacts were seen in some sequences and not in others. It appears that the tray of the MRI device vibrates due to gradient switching, as movement artifacts were only observed in gradient echo sequences (Table 1: T1 3D sequence). The choice of a plastic recipient to maintain the heart in vertical position during MRI examination seemed the easiest and 
Fig. 3 Visualization of coronary arteries with the contrast agent Angiofil ${ }^{\circledR}$ ). a T1-weighted transverse sequence in short axis showing coronary arteries filled with contrast agent (white circles). b MIP (maximumintensity projection) reconstruction of the coronary arteries (white arrows). $R V$ right ventricle, $L V$ left ventricle, $R C A$ right coronary artery, $L C A$ left coronary artery

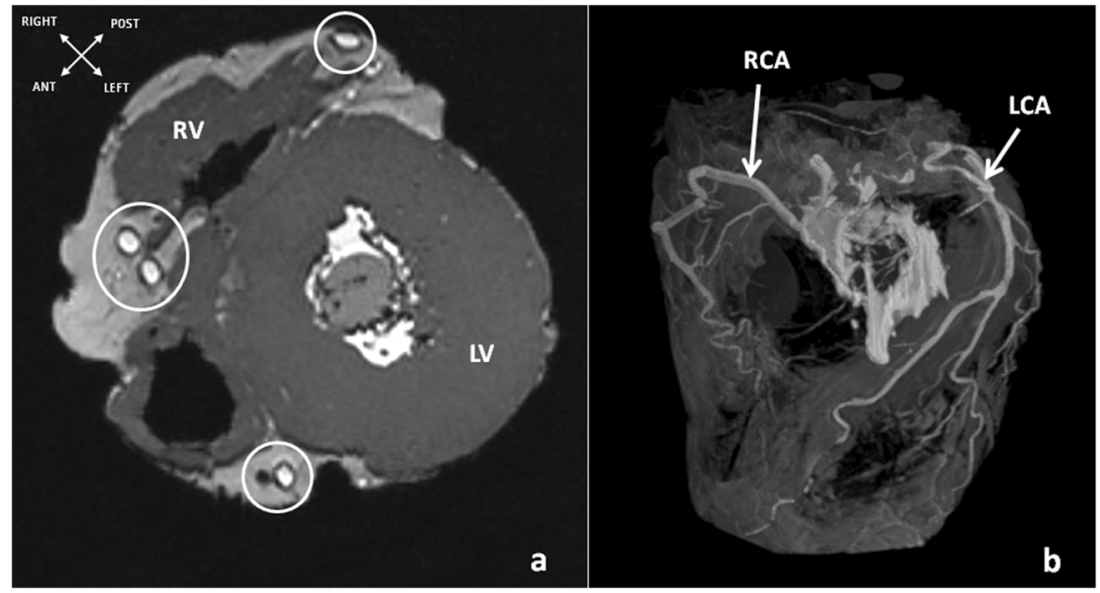

cheapest solution, but this setup was not heavy enough to absorb the vibrations transmitted by the table and led us to increase the weight of the setup. Limited by the size of the container which had to fit in the 32-channel head coil, we tried to fill free spaces around the heart in the container with ordinary sand, but sand contained metallic particles and generated important artifacts (Fig. 4b). We tried decorative sand, which did not generate any artifact, but it is expensive and its density shows great variation according to the provider, causing inconstant weight distribution. The best compromise to create a setup with sufficient weight and justifiable costs was to use a system of two glasses of different diameters with thick bottoms and walls. The two glasses were placed one within the other, and the space between the two glasses was filled with wheat flour. Flour has the advantage of being easy to get, cost efficient, and user-friendly, and its variability of density is negligible. We deliberately did not add water to the setup instead of or in addition to flour, because water MRI signal could interfere with the studied heart MRI signal.
Filling of cardiac cavities

The analysis of cardiac parenchyma required the individualization of the walls of the two ventricles from the interventricular septum. To do this, we attempted to use a small balloon of a urinary catheter, but it generated magnetic susceptibility artifacts (Fig. 4c) impeding the analysis of the myocardium in some sequences. The use of small polystyrene balls avoided these artifacts, as they do not emit any signal. However, their handling is inconvenient because of static electricity. Furthermore, their introduction into the ventricle cavity and their removal are complicated and time consuming. We found modeling clay to be an ideal material providing sufficient stability to keep the ventricle walls separated and is shapeable, permitting its easy introduction into glove fingers or wrapping in cellophane paper, in order to be removed quickly and completely. Modeling clay showed a low signal in T1-weighted and no signal in T2weighted MRI sequences.
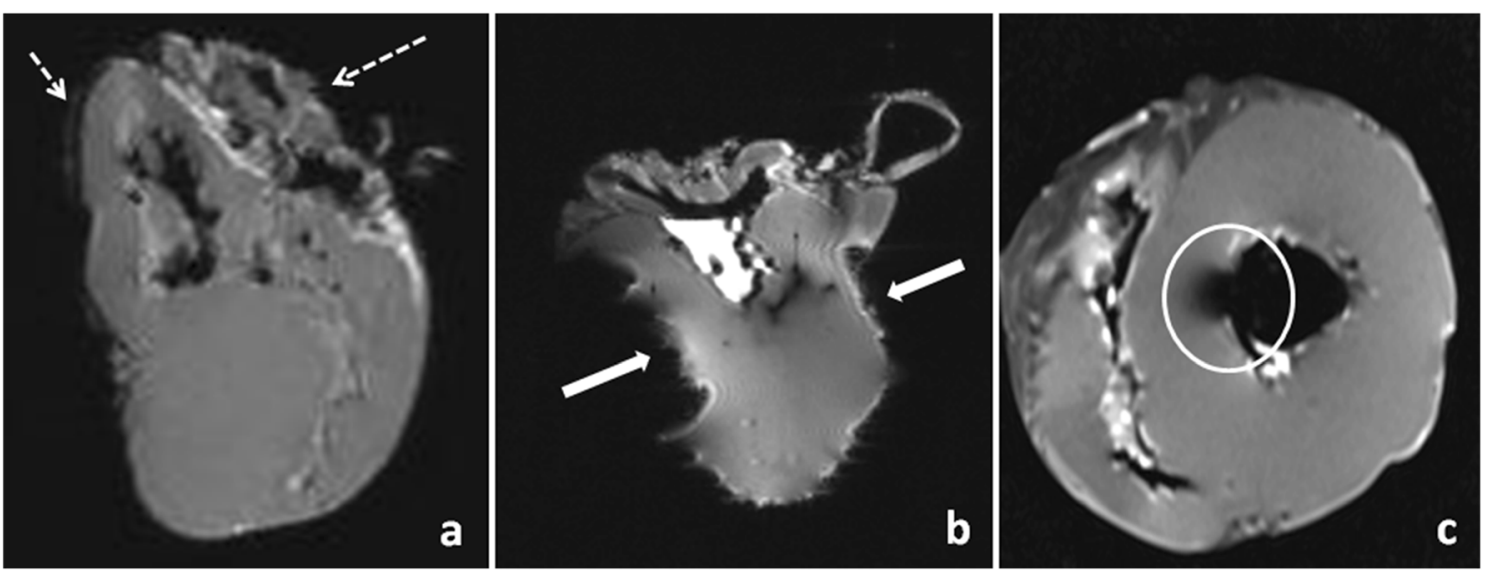

Fig. 4 Various artifacts observed during the testing of different protocols and material. a Movement artifacts (white dashed arrows) caused by vibrations of the MRI table. b Metallic artifacts (white arrows) induced by metallic particles in sand that was used to weight the containers. $\mathbf{c}$
Artifacts of magnetic susceptibility (white circle) caused by a different amplitude of magnetization between the air in a balloon, introduced into cardiac cavities in order to prevent their collapse, and the parenchyma 
Positioning of the heart

As the heart had to be examined ex situ, reproducible positioning was crucial. The simplest way to perform MRI would have been in a horizontal position, but the risk was to there was a risk of compression of the coronary arteries, which could prevent detailed analysis and possibly lead to misinterpretation of atherosclerosis or stenosis. We determined that vertical positioning was the best way to examine the heart ex situ, as it avoided compression of the vessels and allowed for filling of the coronary arteries with contrast agent, "assisted by gravity." Indeed, as explained in the next paragraph, manual filling of the coronary arteries with a catheter was difficult and we observed that filling was better in cases in which we performed an initial instillation of the contrast agent, waited a few minutes with the heart vertically positioned, and then performed a second instillation. On the other hand, this vertical positioning choice generated new problems concerning spatial localization, which was not the same as in daily clinical practice, and the MRI software did not offer the option of choosing an "upright position" in the technical parameters. Moreover, it was difficult to recognize the standard radiological anatomy of the heart without in situ anatomical landmarks. A solution was found with the introduction of a cork stopper into the thoracic ascending aorta and another one in the pulmonary trunk. Cork stoppers had a number of advantages, such as their diameter, which was close to the diameter of the thoracic ascending aorta and the pulmonary trunk, their stiffness, and their MRI-compatible composition. So that we could quickly locate the different structures of the heart, a capsule of vitamin E was introduced into the cork stopper placed in the thoracic ascending aorta. Vitamin E is oily and showed little signal in T1-weighted sequences, and it was easy to differentiate it from the two major vessels (Fig. 2c) and from other structures in the heart. To further facilitate spatial orientation, the heart was always placed into the head coil in the same position, with the anterior side of the heart facing the entrance of the magnet and in the vertical position.

\section{Coronary MR angiography}

A major issue with respect to filling the coronary arteries with contrast agent was to find a catheter that was flexible enough to permit an easy introduction as far as possible into the vessels without tearing them and without inducing atherosclerotic plaque migration. Therefore, we deemed small plastic catheters with a corresponding diameter to be the best choice for instilling contrast agent into the vessels. For our study, we chose cardiac catheter introducers that had passed their expiration date. Even with this innovation, it was difficult to fill the coronary arteries properly, due to their small size and the high viscosity of the contrast agent. Dilution of the oily contrast agent with $50 \%$ paraffinum perliquidum, together with passive vessel filling "assisted by gravity," enabled us to fill the vessels (Fig. 3b).

Our next task was to find a way to maintain the contrast agent in the vessels. Ligature of the coronary arteries near their ostia was not optimal, because of the loss of information concerning the proximal first millimeters of the vessels. However, it was the best procedure we could find. We tried to use a small balloon from a urinary catheter placed in the aorta to plug the ostia from the outside, but since the contrast agent was oily, the balloon could not be maintained in place and the contrast agent flowed out.

\section{Choice of MRI sequences}

When choosing MRI sequences, we relied on clinical practice and experience in the field of cardiac MRI examinations, with the following restrictions: sequences with proton displacements such as time of flight (TOF), dark blood, and late gadolinium enhancement sequences [21, 22] require living patients, cannot be applied to PMMR. Since the MRI device that we used was dedicated to clinical brain research, the software did not contain cardiac-specific sequences. Indeed, some standard clinical sequences, such as proton densityweighted (PD-weighted) sequences, were missing. Moreover, we had to comply with a predetermined time schedule for performing MRI examinations; this limited the examination time to $1 \mathrm{~h}$ per case and compelled us to optimize the choice of sequences. The usefulness of sequences is determined by the indication for the exam, and the specific parameters of the sequences are determined by the chosen coil and the magnitude of the magnetic field; in our case, it was 3 Tesla. Since it is a small organ, ex situ examination of the heart allowed for the use of a 32-channel head coil and thus a small field of view (FOV) of 16-20 cm. Thin slices with a thickness of $0.6-$ $2.5 \mathrm{~mm}$ were chosen, which enabled high-resolution images (Table 1). As in clinical practice, our set of acquisitions contained T1-weighted (Fig. 3a) and T1-weighted fat-saturated sequences (Fig. 5a), which permitted a thorough analysis of the anatomical structures of the myocardium and the coronary arteries. Our set of sequences also contained T2-weighted (Fig. 5b) and T2-weighted fat-saturated sequences (Fig. 5c), which are useful for detecting subsequent edema in cases of myocardial infarction [23-26]. Two sequences were acquired in 3D (T1 3D and T2 Fat/Sat 3D), which allowed for post processing, such as maximum-intensity projection (MIP) (Fig. 3b) and reconstruction of images in different axes (Fig. 6a, b).

All of the sequences were acquired along the short axis of the left ventricle, as in clinical routine; this also correlated with how the organ dissection was performed by the forensic pathologist in our institute. To avoid complicated manipulations, we decided not to perform acquisitions in the right ventricle axis, which is performed in clinical practice (e.g., 


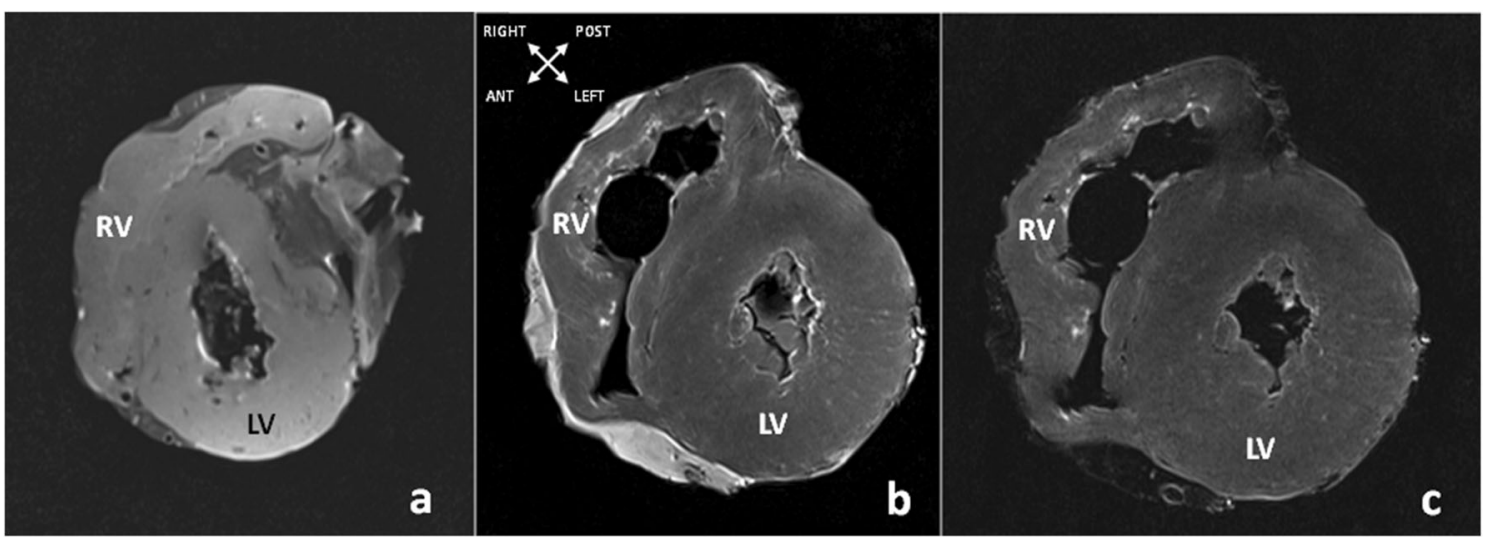

Fig. 5 Acquisitions in short axis of the left ventricle. a T1 fat/sat-weighted sequence. b T2-weighted sequence. c T2 fat/sat-weighted sequence

to detect dysplasia). We used the data from the $3 \mathrm{D}$ sequences to reconstruct images along this axis (Fig. 6a, b). All of the MRI acquisitions were available for analysis by a boardcertified and clinically trained radiologist. Currently, an independent radiological study on a larger number of cases is ongoing in order to evaluate the influence of the postmortem delay and temperature on the image quality.

\section{Limitations of the presented protocol}

Manual filling of the coronary arteries was sometimes suboptimal and completely dependent on the human manipulation. However, to our knowledge, an injector device that would enable filling of the vessels of an ex situ organ, such as the one that is used with the MPMCTA method, does not exist. Another limitation is that it was impossible to analyze the proximal first millimeters of the coronary arteries due to their ligature.

Because we did not have access to an MRI unit with cardiac equipment, the presented scanning protocol was developed while using neurological equipment. Although the principal choice of sequences is transferable to any other MRI unit and any other piece of equipment, it will of course have to be adapted to each unit specifically.
In this study, we did not investigate the influence of the temperature of the examined heart on the quality of the images. As the temperature is an important criterion in MRI, this point has to be clarified in future studies.

Investigation of MRI compatibility of Angiofil ${ }^{\circledR}$

The contrast agent Angiofil ${ }^{\circledR}$ is regularly used in MPMCTA [27]. As this method is routinely used in investigations of cases of suspected natural death due to coronary disease, it was important to test whether it could also be used for MRI investigations, particularly because our center believes that PMMR will eventually be employed after MPMCTA. Therefore, we had to ensure that the contrast agent does not create any artifacts that would contraindicate its application in PMMR. Based on our results, there is no risk of contrastinduced artifacts and Angiofil ${ }^{\circledR}$ is a suitable contrast agent for MRI investigations. This fact can be explained by its composition. As it is an oily liquid, Angiofil ${ }^{\circledR}$ shows a massive fatty signal in T1-weighted sequence, providing contrast to vessel lumens, similar to any clinical MRI contrast agent. This, and the already well-known fact that the oily liquid remains intravascular even hours and days after its injection [28], makes it a suitable contrast agent for ex situ MRI examination of organs.
Fig. 6 Reconstruction of the right ventricle axis. a T1-weighted sequence. b $\mathrm{T} 2 \mathrm{fat} / \mathrm{sat}-$ weighted sequence

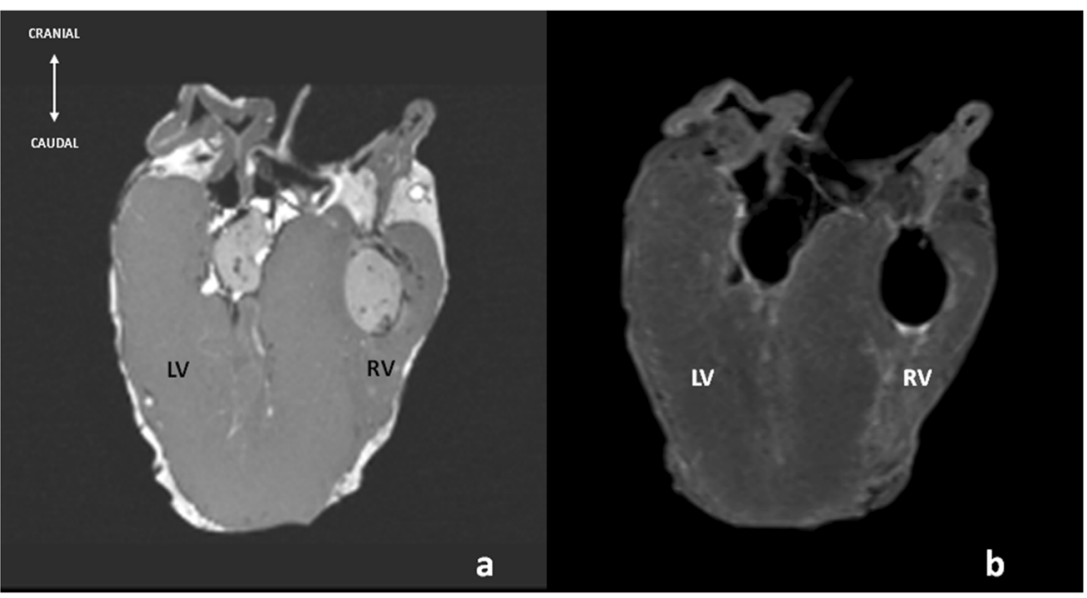


Our experience also showed that preparation of the contrast agent differs slightly when used to examine ex situ organs. For whole-body CT angiography such as MPMCTA, Angiofil ${ }^{\circledR}$ has to be mixed with paraffinum liquidum (Brenntag, Switzerland) [3]. In order to facilitate manual injection of the contrast agent directly into relatively small vessels such as the coronary arteries, we chose to use the less-viscous paraffinum perliquidum to reduce the viscosity of the contrast agent. With this mixture, it was possible to opacify the coronary arteries even in their distal parts. An ongoing radiological study is currently analyzing in detail the quality of the angiograms (filling of the arteries, presence of artifacts, etc.).

\section{Conclusion}

In this paper, we propose a standardized protocol for an ex situ heart preparation procedure that includes coronary MR angiography and PMMR examination of the prepared organ with basic sequences that are routinely used in clinical MRI. This protocol is reproducible, easy to use, and can be applied relatively quickly. We also examined the MRI compatibility of the oily contrast agent Angiofil ${ }^{\mathbb{R}}$, which turned out to be suitable for ex situ coronary MR angiography.

Acknowledgments One of the authors (SG) has personal research funding from the Fondation Leenaards, Lausanne, Switzerland.

The contrast agent Angiofil ${ }^{\mathbb{R}}$ was provided for this study by the Swiss Company Fumedica AG.

The authors thank Mr Pascal Chèvre, radiographer deputy chief of the MRI unit of the Service of Diagnostic and Interventional Radiology form the University Hospital Lausanne, and Bruno Bonet, radiographer in the Brain Behaviour Laboratory of the University of Geneva, for their appreciated help.

Conflict of interests None of the authors have any conflicts of interest to declare.

\section{References}

1. Chevallier C, Doenz F, Vaucher P, Palmiere C, Dominguez A, Binaghi S, Mangin P, Grabherr S (2007) Postmortem computed tomography angiography vs. conventional autopsy: advantages and inconveniences of each method. Int J Leg Med 127:981-989. doi:10. 1007/s00414-012-0814-3

2. Grabherr S, Djonov V, Yen K et al (2007) Postmortem angiography: review of former and current methods. AJR 188:832-838

3. Grabherr S, Doenz F, Steger B, Dirnhofer R, Dominguez A, Sollberger B, Gygax E, Rizzo E, Chevallier C, Meuli R, Mangin P (2010) Multi-phase postmortem CT angiography: development of a standardized protocol. Int J Med Leg 125:791-802

4. Palmiere C, Binaghi S, Doenz F, Bize P, Grabherr S (2012) Detection of hemorrhage source: the diagnostic value of postmortem CT angiography. Forensic Sci Int 222:33-39

5. Michaud K, Grabherr F, Doenz MP (2012) Evaluation of postmortem MDCT and MDCT-angiography for the investigation of sudden cardiac death related to atherosclerotic coronary artery disease. Int $\mathrm{J}$ Cadiovasc Imaging 28:1807-1822

6. Friedrich MG (2008) Tissue characterization of acute myocardial infarction and myocarditis by cardiac magnetic resonance. JACC Cardiovasc Imaging 1:652-662

7. Amano Y, Tachi M, Tani H, Mizuno K, Kobayashi Y, Kumita S (2012) T2-weighted cardiac magnetic resonance imaging of edema in myocardial diseases. Scientific World Journal 194069

8. Ruder TB, Hatch GM, Ebert LC, Flach PM, Ross S, Ampanozi G, Thali MJ (2012) Whole body postmortem magnetic resonance angiography. J Forensic Sci 57:778-782

9. Jachau K, Heinrichs T, Kuchheuser W, Krause D, Wittig H, Schöning R, Beck N, Beuing O, Döhring W, Jackowski C (2004) CT- und MRT-Befunde an isolierten Leichenhezen. Rechtsmedizin 14:109 116. doi:10.1007/s00194-004-0252-8

10. Jackowski C, Schweitzer W, Thali M, Yen K, Aghayev E, Sonnenschein M, Vock P, Dirnhofer R (2005) Virtopsy: postmortem imaging of the human heart in situ using MSCT and MRI. Forensic Sci Int 149:11-23

11. Jackowski C, Hofmann K, Schwendener N, Schweitzer W, KellerSutter M (2012) Coronary thrombus and peracute myocardial infarction visualized by unenhanced postmortem MRI prior to autopsy. Forensic Sci Int 214(1-3):e16-e19

12. Ruder TD, Bauer-Kreutz R, Ampanozi G, Rosskopf AB, Pilgrim TM, Weber OM, Thali MJ, Hatch GM (2012) Assessment of coronary artery disease by postmortem cardiac MR. Eur J Radiol 81: $2208-2214$

13. Jackowski C, Thali M, Aghayev E, Yen K, Sonnenshein M, Zwygart K, Dirnhofer R, Vock P (2006) Postmortem imaging of blood and its characteristics using MSCT and MRI. Int J Leg Med 120:233-240

14. Jackowski C, Christe A, Sonnenschein M, Aghayev E, Thali MJ (2006) Postmortem unenhanced magnetic resonance imaging of myocardial infarction in correlation to histological infarction age characterization. Eur Heart J 27:2459-2467. doi:10.1093/eurheartj/ ehl255

15. Jackowski C, Schwendener N, Grabherr S, Persson A (2013) Postmortem cardiac 3-T magnetic resonance imaging: visualization of sudden cardiac death? J Am Coll Cardiol 13(62):617-629

16. Heidenreich PA, Trogdon JG, Khavjou OA, Butler J, Dracup K, Ezekowitz MD, Finkelstein EA, Hong Y, Johnston SC, Khera A, Lloyd-Jones DM, Nelson SA, Nichol G, Orenstein D, Wilson PW, Woo YJ (2011) Forecasting the future of cardiovascular disease in the United States: a policy statement from the American Heart Association. Circulation 123:933-944

17. Roberts ISD, Benbow EW, Bisset R, Jenkins JPR, Lee SH, Reid H, Jackson A (2003) Accuracy of magnetic resonance imaging in determining cause of sudden death in adults: comparison with conventional autopsy. Histopathology 42:424-430

18. Anonymous (2000) Recommendation no R(99)3 of the committee of ministers to member states on the harmonization of medico-legal autopsy rules. Council of Europe, Committee of Ministers. Adopted by the Committee of Ministers on 2 February 1999 at the 658th meeting of the Ministers' Deputies

19. Brinkmann B (1999) Harmonisation of medico-legal autopsy rules. Int J Leg Med 113:1-14

20. Florian A, Jurcut R, Ginghina C, Bogaert J (2011) Cardiac magnetic imaging in ischemic heart disease: a clinical review. J Med Life 4: 330-345

21. Kellman P, Mancini C, Vasu S, Leung SW, Wilson JR, Shanbhag SM, Chen MY, Arai AE (2012) MultiContrast Delayed Enhancement (MCODE) improves detection of subendocardial myocardial infarction by late gadolinium enhancement cardiovascular magnetic resonance: a clinical validation study. Cardiovasc Magn Reson 14:83

22. Srichai MB, Lim RP, Lath N, Babb J, Axel L, Kim D (2013) Diagnostic performance of dark-blood T2-weighted CMR for evaluation of acute myocardial injury. Invest Radiol 48:24-31 
23. Abdel-Aty H, Coker M, Tyberg JV, Friedrich MG (2009) Edema as a very early marker for acute myocardial ischemia: a cardiovascular magnetic resonance study. J Am Cardiol 53:1194-1201

24. Carbone I, Friedrich MG (2012) Myocardial edema imaging by cardiovascular magnetic resonance: current status and future potential. Curr Cardiol Rep 14:1-6

25. Abdel-Aty H, Tillmanns C (2010) The use of cardiovascular magnetic resonance in acute myocardial infarction. Curr Cardiol Rep 12: $76-81$
26. Abdel-Aty H, Schulz-Menger J (2007) Cardiovascular magnetic resonance T2-weighted imaging of myocardial edema in acute myocardial infarction. J Recent Pat Cadiovasc Drug Discov 2:63-68

27. Grabherr S, Grimm J, Dominguez A, Vanhaebost J, Mangin P (2014) Advances in post-mortem CT-angiography. Br J Radiol Jan 7 [Epub ahead of print]

28. Grabherr S, Djonov V, Friess A, Thali MJ, Ranner G, Vock P, Dirnhofer R (2006) Postmortem angiography after vascular perfusion with diesel oil and a lipophilic contrast agent. AJR 187:W515-W523 